Article

\title{
Personal Picocell Scheme Using Adaptive Control CRE in Heterogeneous Mobile Networks ${ }^{+}$
}

\author{
Kento Fujisawa ${ }^{1}$, Fumiya Kemmochi ${ }^{1}$ and Hiroyuki Otsuka ${ }^{2, *}$ \\ 1 Graduate School of Engineering, Kogakuin University, Tokyo 163-8677, Japan; \\ cm19040@ns.kogakuin.ac.jp (K.F.); cm19016@ns.kogakuin.ac.jp (F.K.) \\ 2 Department of Information and Communications Engineering, Kogakuin University, Tokyo 163-8677, Japan \\ * Correspondence: otsuka@cc.kogakuin.ac.jp \\ $+\quad$ This paper is an extended version of a paper published in International Conference on 90th Vehicular \\ Technology Conference (VTC2019-Fall), entitled "Personal-Cell Scheme Using Adaptive Control CRE for \\ Multicarrier HetNets", ISBN 978-1-7281-1220-6, doi:10.1109/VTCFall.2019.8891193, published by IEEE.
}

Received: 17 August 2020; Accepted: 10 October 2020; Published: 14 October 2020

check for updates

\begin{abstract}
Heterogeneous networks (HetNets), which are combined with a macrocell and picocell in the same coverage, are expected to further increase the system capacity in fifth-generation mobile systems and beyond. In HetNets, the cell range expansion (CRE) technique plays an important role and can allow more user equipment (UE) to access the picocell, i.e., virtually expand the picocell coverage. However, conventional CRE techniques that provide a fixed cell selection offset (CSO) for all UE may worsen user throughput if UE is forced to connect to the picocell because the received signal-to-interference plus noise ratio of the UE becomes lower. Therefore, we propose a personal picocell scheme using an adaptive control CRE technique to improve user throughput in which different CSOs are assigned to UE to form each optimal picocell for each UE. In this paper, we first describe the aspects and algorithm of the proposed scheme. Then, we show the user throughput for adaptive control CRE in comparison with conventional CRE by using system-level computer simulations for the two types of HetNets, i.e., single-band and multi-band HetNets. In the simulations, we first clarify the optimal parameters of the adaptive control CRE. We then show the average and 5-percentile user throughput of the optimized adaptive control CRE in comparison with that of conventional CRE. From these results, we confirmed that the personal picocell scheme using the adaptive control CRE can improve the 5-percentile user throughput while maintaining the average user throughput compared with that of conventional CRE.
\end{abstract}

Keywords: mobile communication; heterogeneous networks; cell range expansion; cell selection offset; personal picocell scheme; user throughput

\section{Introduction}

Fifth-generation (5G) mobile systems are being developed, notably in the third-generation partnership project (3GPP) standards body, and have been partially launched worldwide, and the 5G coverage area is gradually being expanded. The primary objectives of $5 \mathrm{G}$ are to increase system capacity and improve data rates, which is called enhanced mobile broadband (eMBB), and achieve ultra-reliable low latency communication (URLLC) while providing massive machine type communications (mMTC) based on the Internet of Things (IoT) [1-5].

Two type of 5G systems are approved by the 3GPP standards body; one is 5G New Radio (NR) with non-standalone (NSA) operation which enables 5G NR deployments using existing fourth-generation (4G) mobile systems, such as long-term evolution (LTE). Another one is 5G NR with standalone (SA) operation, which will provide a complete 5G NR with a 5G core network. The initial phase of 
5G NR NSA focuses on eMBB using both 5G NR and 4G LTE. URLLC and mMTC are expected to be provided by the use of 5 G NR SA, although $\mathrm{mMTC}$ has already been developed using 3GPP release 13 low power wide area technology, such as NB-IoT.

From the network-density perspective, small cell strategy and heterogeneous network (HetNet) approaches are important for increasing system capacity and/or for improving data rates. The small cell approach is a straightforward method in which each low-power evolved node B (eNB) in the small cell is directly connected to a core mobile network. This approach can increase the system capacity compared with traditional large-scale macrocell networks because system capacity, i.e., radio resources, is proportional to the number of small cells. Considering control channel operation such as handover for user equipment (UE), the concept of a phantom cell has also been proposed in which the control and user-plane are separated within the same coverage [6]. Consequently, the macro-eNB manages all UE within the overlaid cells, although the UE can connect to a neighboring low-power eNB in a small cell.

The HetNet approach is also one of the denser mobile networks, which is comprised of a combination of different cell types [7-11]. For example, picocells with low-power eNBs are installed within the coverage area of a macrocell, and the purpose is to allow UE to access the picocells even though the UE is within the donor macrocell. Consequently, HetNet can increase the system capacity, especially when the traffic in the macro cell enormously increases.

One important issue in HetNet deployments is to ensure that the picocells serve enough UE. One way to do that is the cell range expansion (CRE) technique, which can virtually expand the picocell coverage through the use of a positive cell selection offset (CSO) for the downlink reference-signal received power (RSRP) from the picocell [12-14]. In a normal case without CRE, the cell selection is carried out for UE based on the comparison between the downlink RSRPs from the macrocell and picocell. With CRE, a positive CSO is added to the downlink RSRP from the picocell to expand the coverage served by the picocell. Therefore, CRE can allow more UE to access the picocell.

However, the CRE technique has one drawback in that the downlink RSRP of UE that is forcibly served by the picocell located in the CRE zone is lower because the downlink RSRP from the macrocell is in reality higher than that from the picocell. Consequently, the user throughput of UE served by the picocell in the CRE zone may worsen.

Based on this background, to overcome this drawback, we have proposed a personal picocell scheme using adaptive control CRE that can provide an optimal different CSO for each piece of UE [15-20]. For example, in ref. [15], we presented user throughput in co-channel (single-band) HetNets using a $2.0 \mathrm{GHz}$ band by means of a modulation and coding scheme (MCS) up to a 64-quadrature amplitude modulation (QAM). In ref. [20], we presented user throughput in multi-band HetNets using 2.0 and $3.4 \mathrm{GHz}$ by means of a MCS up to 256-QAM.

In this study, we evaluate user throughput of adaptive control CRE comprehensively for both single- and multi-band HetNets under the same MCS condition, i.e., MCS up to 256-QAM [21-24]. The key contributions of this work are summarized as follows.

- We propose a new concept of a personal picocell scheme in HetNet and propose an adaptive control CRE technique to realize the personal picocell scheme through the use of two different CSOs.

- We first clarify the optimal parameters of the proposed adaptive control CRE for single- and multi-band HetNets. We then show the average and 5-percentile user throughput of the optimized adaptive control CRE and compare them with those of conventional CRE.

This paper is organized as follows. In Section 2, we introduce the concept and architecture of the personal picocell scheme and adaptive control CRE using two different CSOs. In Section 3 , we discuss the parameter optimization of the adaptive control CRE, and show the user throughput of the optimized adaptive control CRE in single-band HetNet in comparison with the conventional method. In Section 4, we show the user throughput of the optimized adaptive control CRE in a multi-band HetNet. Finally, we conclude our work in Section 5. 


\section{Personal Picocell Scheme Using Adaptive Control CRE}

\subsection{HetNets and CRE}

Figure 1 shows a typical HetNet combined with a three-sector macrocell served by a macro-eNB and picocell for each macro-sector served by each pico-eNB. Picocells are primarily added to increase the system capacity in hot spots with considerable UE, including indoor areas. By offloading traffic from the macrocell, HetNet can improve the system performance, i.e., provide increased user throughput.

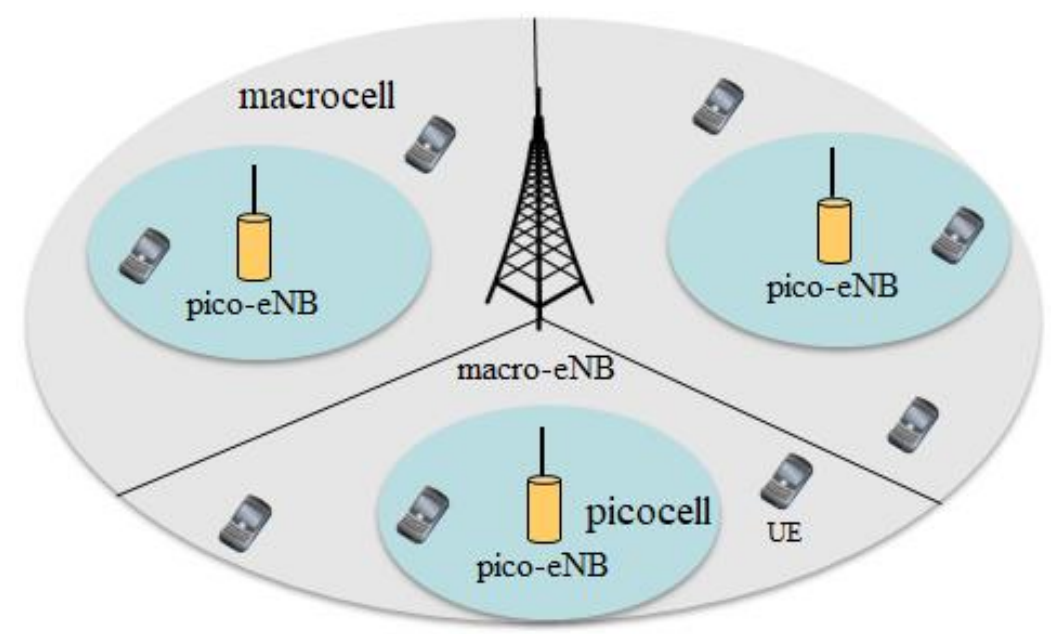

Figure 1. HetNet combined with macrocell served by macro-eNB and picocell served by pico-eNB [20].

From a carrier frequency perspective, two types of HetNets have been discussed, as shown in Figure 2. The first is single-band HetNet in which the carrier frequency of the picocell is the same as that of the macrocell, as shown in Figure 2a. The second is multi-band HetNet in which the carrier frequencies between the macrocell and picocell are different, as shown in Figure $2 b$. The single-band HetNet causes interference between the macrocell and picocell, as well as interference between picocells. However, multi-band HetNet causes interference only between picocells. In this paper, a HetNet with time-domain enhanced inter-cell interference coordination (eICIC) to eliminate the interference between the macrocell and picocell is not considered [19].

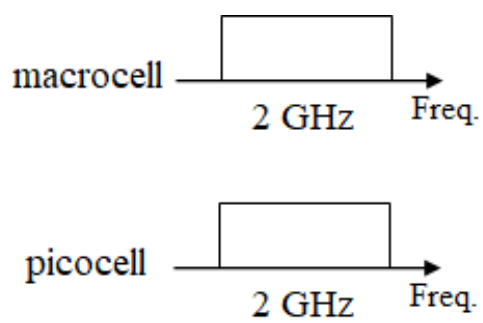

(a)

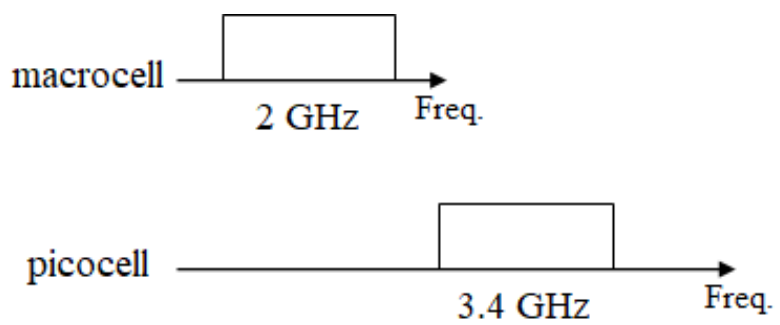

(b)

Figure 2. Two types of HetNets. (a) Single-band HetNet; (b) multi-band HetNet.

In HetNets, it is important for UE to determine which access is better to communicate with the macro- or pico-eNBs, which is referred to as cell selection. UE is normally connected to a cell with a stronger downlink RSRP or with a higher signal-to-interference plus noise ratio (SINR). Therefore, the connection ratio of the macro-eNB is greater than that of the pico-eNB because the transmission power of the macro-eNB is larger than that of the pico-eNB. CRE technique is very effective at increasing the connection ratio of the pico-eNB through the use of a positive CSO for the downlink RSRP from the pico-eNB, as shown in Figure 3, although the downlink transmission power of 
the pico-eNB is fixed (i.e., unchanged for UE). In this case, the picocell coverage appears to be wider as an "extended picocell" for the UE in the CRE zone, as shown in Figure 3. Consequently, the connection point of the UE in the CRE zone should be changed from macro-eNB to pico-eNB when CRE is activated. Like this, CRE technique is effectively used in HetNets to enhance the system capacity as well as cell-edge user throughput. However, CRE degrades the downlink RSRP of the UE that is forcibly served by the pico-eNB located in the CRE zone because the downlink RSRP from the macro-eNB is in reality higher than that from the pico-eNB. According to the CRE drawback, the user throughput of the UE that is served by the pico-eNB in the CRE zone may worsen.

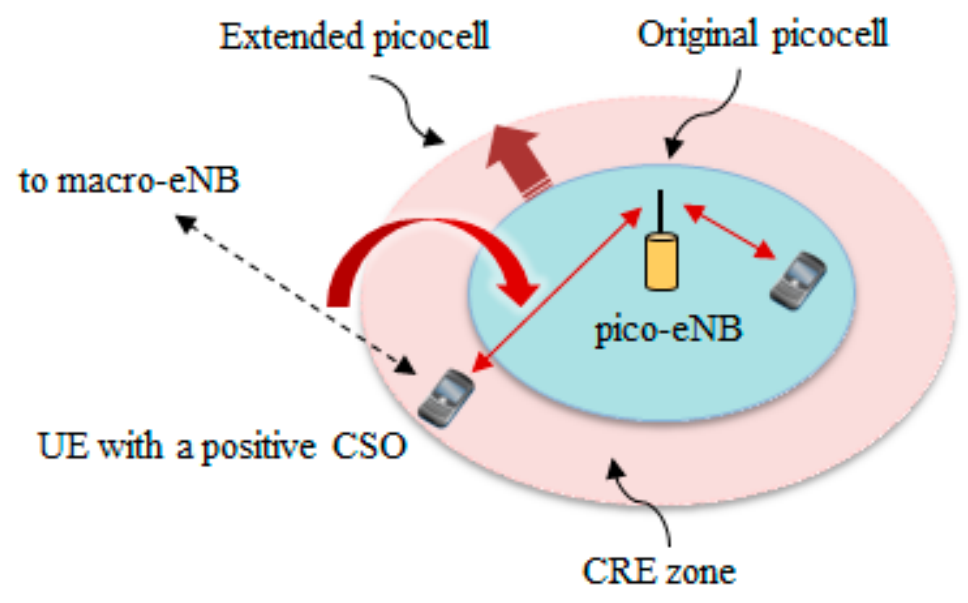

Figure 3. Principle of cell range expansion (CRE) with a positive cell selection offset (CSO) in HetNet.

Interference scenario for pico-eNB installed at outdoor is different from that for indoor pico-eNB. Therefore, the usefulness of CRE is scenario dependent. For example, the CRE may not be useful for indoor pico-eNB, because buildings wall provide isolation between the microcell and picocell.

From the uplink performance perspective, CRE would increase the uplink received power and/or SINR at pico-eNB when the connection point is changed to the pico-eNB from macro-eNB by the CRE. It is well-known that uplink cell border is different from the downlink border in HetNets. In general, the uplink border is closer to macro-eNB compared with the downlink border. Therefore, the received SINR at pico-eNB for UE within CRE zone can be improved, because the path-loss to the pico-eNB is less than that to the macro-eNB.

\subsection{Proposal of Personal Picocell Scheme}

In conventional CRE technology, a fixed CSO is applied to all UE within the macrocell or macro-sector. However, as mentioned in Section 2.1, CRE actually induces a decreased downlink RSRP for the UE in the CRE zone. Because the received downlink SINR of the UE from the pico-eNB decreases, the throughput of the UE must be lower unless the radio resource assignment is unchanged. However, CRE can be expected to increase the throughput because the pico-eNB can assign more radio resources to the UE in the CRE zone.

Therefore, to solve this problem, we propose a personal picocell scheme in which the picocell coverage should be independent of each UE, i.e., the CRE zone should be independent of each UE when the CRE is activated on the condition that the downlink transmission power of the pico-eNB is fixed [18]. The basic concepts are to provide a positive high CSO for UE with lower downlink SINR from macro-eNB to encourage the connection to pico-eNB, and conversely to provide a positive low CSO for UE with higher downlink SINR to maintain the connection to macro-eNB. Figure 4 shows an example of a personal picocell scheme in which CRE can form an independent extended picocell: "a picocell for $\mathrm{UE}_{1}$ with a $\mathrm{CSO}_{1}$ around pico-eNB${ }_{1}$ " and "a picocell for $\mathrm{UE}_{2}$ with a $\mathrm{CSO}_{2}$ around pico-eNB 2 ." Similarly, $\mathrm{CRE}$ for pico-eNB 3 can also form an independent extended picocell, "a picocell 
for $\mathrm{UE}_{3}$ with a $\mathrm{CSO}_{3}$ " and "a picocell for $\mathrm{UE}_{4}$ with a $\mathrm{CSO}_{4}$," even though $\mathrm{UE}_{3}$ and $\mathrm{UE}_{4}$ are connected to the same pico-eNB 3 .

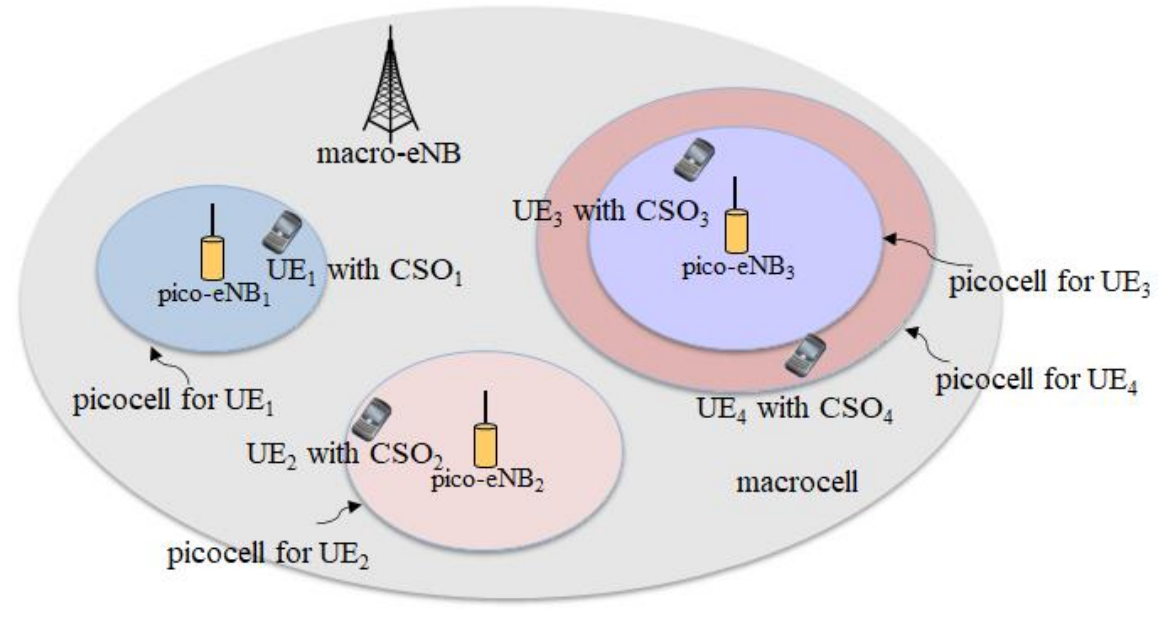

Figure 4. Concept of personal picocell scheme in HetNet.

\subsection{Adaptive Control CRE}

In general, cell selection is executed using downlink RSRP which is represented as Equation (1).

$$
j_{\mathrm{u}}=\arg \max P_{\mathrm{i}, \mathrm{u}}
$$

where, $i$ and $u$ show the cell ID and user ID (UE ID), respectively. $j_{u}$ is the donor cell ID that is chosen by $\mathrm{UE}_{\mathrm{u}}$, and $P_{\mathrm{i}, \mathrm{u}}$ represents the RSRP at $\mathrm{UE}_{\mathrm{u}}$ obtained from the reference signal of cell-i. CRE is basically activated using RSRP-based method. According to CRE, UE makes an offset to the RSRP obtained from the reference signal of the picocell, which is given by Equations (2) and (3).

$$
\begin{gathered}
j_{\mathrm{u}}=\arg \max \left(P_{\mathrm{i}, \mathrm{u}}+\gamma_{\mathrm{i}}\right) \\
\gamma_{\mathrm{i}}=\left\{\begin{array}{cc}
\mathrm{CSO} ; & \text { if } \mathrm{i} \text { is a picocell ID } \\
0 ; & \text { if } \mathrm{i} \text { is a macrocell ID }
\end{array}\right.
\end{gathered}
$$

where, $\gamma_{\mathrm{i}}$ represents the CSO which is applied to UE located within and/or close to cell-i. If CRE does not work, i.e., $\mathrm{CSO}=0 \mathrm{~dB}$, the picocell coverage remains unchanged. CRE is not applied for microcell; therefore, $\gamma_{\mathrm{i}}$ is fixed to 0 .

As mentioned in Section 2.2, it could be ideal for each UE to provide each CSO to realize the personal picocell scheme. However, it would be impossible to do that in real mobile networks because the number of UE is enormous. Therefore, we propose an adaptive control CRE that uses two different $\mathrm{CSO}\left(\mathrm{CSO}_{\text {high }}\right.$ and $\left.\mathrm{CSO}_{\text {low }}\right)$. In the proposed method, $\mathrm{CSO}$ is given by Equation (3).

$$
\gamma_{\mathrm{i}, \mathrm{u}}=\left\{\begin{array}{cc}
\mathrm{CSO}_{\text {high }} ; & \text { if } \mathrm{SINR}_{\mathrm{u}}<\mathrm{SINR}_{\mathrm{th}} \\
\mathrm{CSO}_{\text {low }} ; & \text { otherwise }
\end{array}\right.
$$

where $\gamma_{\mathrm{i}, \mathrm{u}}$ indicates the CSO which is applied to $\mathrm{UE}_{\mathrm{u}}$ located near the picocell-i. SINR $\mathrm{u}$ is the downlink SINR measured at the $\mathrm{UE}_{\mathrm{u}}$ obtained from the reference signal of the donor microcell. $\mathrm{SINR}_{\mathrm{th}}$ is assumed to meet a fixed value of $\alpha$ for cumulative distribution function (CDF), where the CDF is formed by the downlink SINR $\mathrm{u}_{\mathrm{u}}$ measured at all UE obtained from the reference signal of macrocell.

Figure 5 shows the principle of the adaptive control CRE and how to determine the two different CSOs. The macro-eNB first determines $\alpha, \mathrm{SINR}_{\text {th }}$ and two different $\mathrm{CSO}\left(\mathrm{CSO}_{\text {high }}\right.$ and $\left.\mathrm{CSO}_{\text {low }}\right)$ based on the results of the CDF. Then, the macro-eNB commands all UE within the macrocell in which $\mathrm{CSO}_{\text {high }}$ is assigned to UE with a SINR lower than $\mathrm{SINR}_{\mathrm{th}}$, and $\mathrm{CSO}_{\text {low }}$ is assigned to UE with a SINR greater 
than $\mathrm{SINR}_{\mathrm{th}}$. Figure 5 also shows the difference in picocell coverage for $\mathrm{UE}_{1}$ and $\mathrm{UECSO}_{\mathrm{high}}$ is assigned to $\mathrm{UE}_{1}$ because $\mathrm{SINR}_{1}$ of $\mathrm{UE}_{1}$ is lower than $\mathrm{SINR}_{\text {th }}$. Similarly, $\mathrm{CSO}_{\text {low }}$ is assigned to $\mathrm{UE}_{2}$ because $\mathrm{SINR}_{2}$ of $\mathrm{UE}_{2}$ is greater than $\mathrm{SINR}_{\mathrm{th}}$. Consequently, the picocell coverage of $\mathrm{UE}_{1}$ looks wider than that of $\mathrm{UE}_{2}$. Figure 6 shows the algorithm of the adaptive control CRE. The fundamental concept for the offloading scenario is the almost same as that for the conventional CRE. The difference is that UE can choose two different $\mathrm{CSO}\left(\mathrm{CSO}_{\text {high }}\right.$ and $\left.\mathrm{CSO}_{\text {low }}\right)$ depending on their downlink received SINR performance.

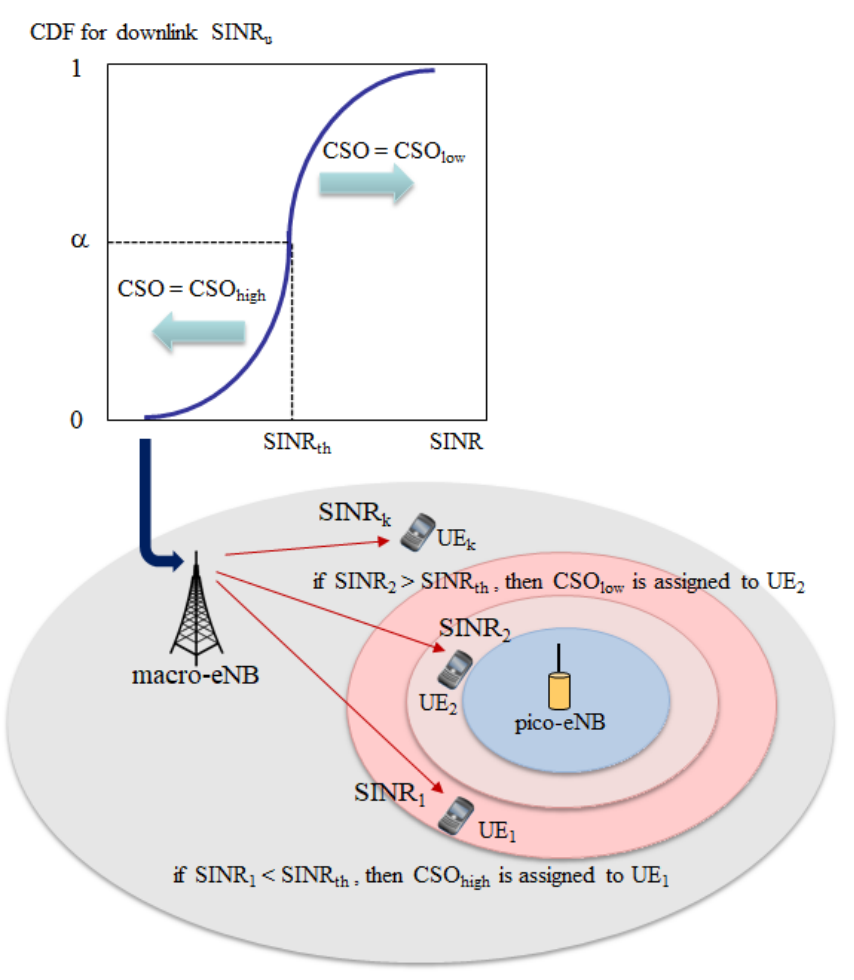

Figure 5. Adaptive control CRE using two different CSOs: $\mathrm{CSO}_{\text {high }}$ and $\mathrm{CSO}_{\mathrm{low}}$.

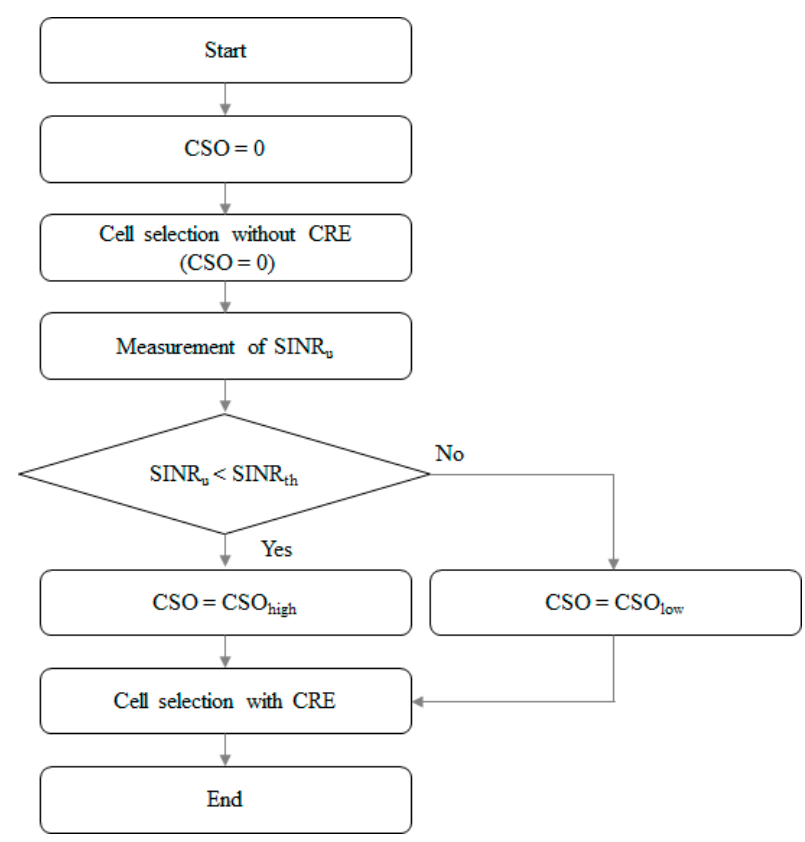

Figure 6. Algorithm of adaptive control CRE in HetNet. 


\section{User Throughput in Single-Band HetNet}

\subsection{Simulation Setup}

In this study, we evaluate the user throughput of adaptive control CRE using system-level computer simulations under the conditions that the number of macrocells with three sectors per site is 19 and the number of picocells is four per macro-sector. The primary simulation parameters are listed in Table 1. The carrier frequencies used in the macro- and pico-eNBs are $2.0 \mathrm{GHz}$. The system bandwidth of both the macro- and pico-eNBs are assumed to be $10 \mathrm{MHz}$. The number of UEs is set to 30 per macro sector. UE is located based on a cluster distribution in which $2 / 3$ of the number of UEs are uniformly deployed nearby the pico-eNBs and the rest (1/3 of the number of UEs) are uniformly deployed within the macrocell. The transmission power in the downlink is fixed at +46 and $+30 \mathrm{dBm}$ for the macro- and pico-eNBs, respectively. The path-loss model and other assumptions are obtained from in ref. [25]. In this paper, we focus on the performance evaluation of downlink user throughput.

Table 1. Simulation parameters [20].

\begin{tabular}{|c|c|c|}
\hline \multirow{2}{*}{ Parameter } & \multicolumn{2}{|l|}{ Value } \\
\hline & Macro-eNB & Pico-eNB \\
\hline Cell layout & Hexagonal grid, 19 cell sites, 3 sectors per site & 4 picos per sector \\
\hline Cell radius (ISD) & $289 \mathrm{~m}(500 \mathrm{~m})$ & 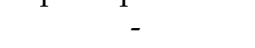 \\
\hline Tx power & $46 \mathrm{dBm}$ & $30 \mathrm{dBm}$ \\
\hline Tx antenna gain & $14 \mathrm{dBi}$ & $5 \mathrm{dBi}$ \\
\hline Antenna height & $32 \mathrm{~m}$ & $10 \mathrm{~m}$ \\
\hline Carrier frequency & $2.0 \mathrm{GHz}$ & 2.0 or $3.4 \mathrm{GHz}$ \\
\hline System bandwidth & $10 \mathrm{MHz}$ & $10 \mathrm{MHz}$ \\
\hline UE distribution & \multicolumn{2}{|c|}{30 UEs per sector, $2 / 3$ clustered distribution } \\
\hline Scheduling algorithm & \multicolumn{2}{|c|}{ Proportional fairness } \\
\hline Link adaptation & \multicolumn{2}{|c|}{15 MCS (QPSK to 256-QAM) } \\
\hline Traffic model & \multicolumn{2}{|c|}{ Full buffer } \\
\hline Link to system mapping & \multicolumn{2}{|l|}{ EESM } \\
\hline MIMO & \multicolumn{2}{|l|}{$2 \times 2$ SU-MIMO } \\
\hline
\end{tabular}

As the use of higher-order QAM is expected to increase data rates within a limited bandwidth, the implementation of 1024-QAM and 4096-QAM has been discussed [26-28] for 5G and beyond. However, in the simulations, we use 15 types of MCS indexes up to 256-QAM in the downlink as listed in Table 2, because the application conditions for these post 256-QAM are currently very limited. This is the same as the channel quality indicator (CQI) indexes specified by 3GPP release 12 . The MCS used in the downlink for UE is determined by the channel quality between the macro-eNB and the UE. The lowest and highest modulation schemes are quadrature phase shift keying (QPSK) and 256-QAM, respectively. MCS index incorporating a higher-order QAM is assigned to UE with good channel quality; however, QPSK is almost assigned to UE with poor channel quality.

System-level simulations are worth evaluating user throughput for mobile network, and will contribute significantly to the requirements at early stages of the development and hardware implementation.

\subsection{Parameter Optimization in Single-Band HetNet}

We first determine the parameter optimization, (i.e., the best combination of $\alpha, \mathrm{CSO}_{\text {high, }}$ and $\mathrm{CSO}_{\text {low }}$ ), of adaptive control CRE to provide the best average and 5-percentile user throughput. Here, the 5-percentile user throughput is defined as the 5th percentile point of the CDF of user throughput. Figure 7a-d shows the average and 5-percentile user throughput versus $\mathrm{CSO}_{\text {high }}$ as a function of $\mathrm{CSO}_{\text {low }}$ for $\alpha=0.2,0.4,0.6$, and 0.8 , respectively. As shown in Figure 7a,b, the performance of $\alpha=0.2$ and 0.4 is better than that of $\alpha=0.6$ and 0 .When $\alpha=0.2$ and 0.4 , a lower $\mathrm{CSO}_{\text {low }}$ provide a better average user throughput and a higher $\mathrm{CSO}_{\text {low }}$ provide a better 5-percentile user throughput. Considering both the average and 5-percentile 
user throughput, the parameters of $\alpha=0.4, \mathrm{CSO}_{\text {low }}=4 \mathrm{~dB}$, and $\mathrm{CSO}_{\text {high }}=6 \mathrm{~dB}$ are determined to be the best.

Table 2. Modulation and coding scheme (MCS) table in downlink [20].

\begin{tabular}{cccc}
\hline MCS Index & Modulation & Coding Rate & Efficiency $(\mathbf{b p s} / \mathbf{H z})$ \\
\hline 0 & & Out of range \\
\hline 1 & & $1 / 13$ & 0.152 \\
2 & QPSK & $1 / 5$ & 0.377 \\
3 & & $7 / 16$ & 0.875 \\
\hline 4 & & $3 / 8$ & 1.500 \\
5 & 16-QAM & $1 / 2$ & 2.000 \\
6 & & $3 / 5$ & 2.400 \\
\hline 7 & & $5 / 9$ & 3.333 \\
8 & & $9 / 20$ & 3.700 \\
9 & 64-QAM & $13 / 20$ & 3.900 \\
10 & & $3 / 4$ & 4.500 \\
11 & & $17 / 20$ & 5.100 \\
\hline 12 & & $7 / 10$ & 5.600 \\
13 & & $19 / 24$ & 6.333 \\
14 & & $13 / 15$ & 6.933 \\
15 & $256-Q A M$ & $23 / 25$ & 7.360 \\
\hline
\end{tabular}

\subsection{Comparison with Conventional Method}

Figure 8 shows the average and 5-percentile user throughput of the proposed adaptive control CRE using the optimal parameters determined in Section 3.2 compared with those for the conventional method and homogeneous network (HomoNet).

In the conventional method, six kinds of fixed CSO are set: $\mathrm{CSO}=0,2,4,6,8$, and $10 \mathrm{~dB}$. The proposed method (Proposal) refers to the use of adaptive control CRE with the parameters of $\alpha=0.4, \mathrm{CSO}_{\text {low }}=4 \mathrm{~dB}$, and $\mathrm{CSO}_{\text {high }}=6 \mathrm{~dB}$. The blue bar shows the average user throughput corresponding to the left-side axis, and the orange bar shows the 5-percentile user throughput corresponding to the right-side axis.

The average user throughput decreases according to an increase of the CSO from 0 to $10 \mathrm{~dB}$. One reason for this phenomenon is the increase of the number of UE in the CRE zone whose user throughput may be degraded. By increasing the CSO up to $4 \mathrm{~dB}$, the 5-percentile user throughput increases, although the average user throughput decreases. One reason is that UE with poor user throughput connected to macro-eNB is connected to pico-eNB. This results in the improvement of the user throughput of the UE. However, a higher CSO decreases the 5-percentile user throughput in the conventional CRE. Likewise, if we attempt to improve the 5-percentile user throughput using a fixed CSO, the conventional CRE causes a deterioration in the average user throughput. However, the adaptive control CRE can solve such kinds of trade-off problems, i.e., improve the 5-percentile user throughput by $5 \%$ while maintaining the average user throughput compared with those of conventional method (a CSO of $4 \mathrm{~dB}$ ), as shown in Figure 8. Compared with a $\mathrm{CSO}$ of $0 \mathrm{~dB}$, the proposed method can improve 5-percentile user throughput by 1.5 times approximately, although the average user throughput slightly decreases. 

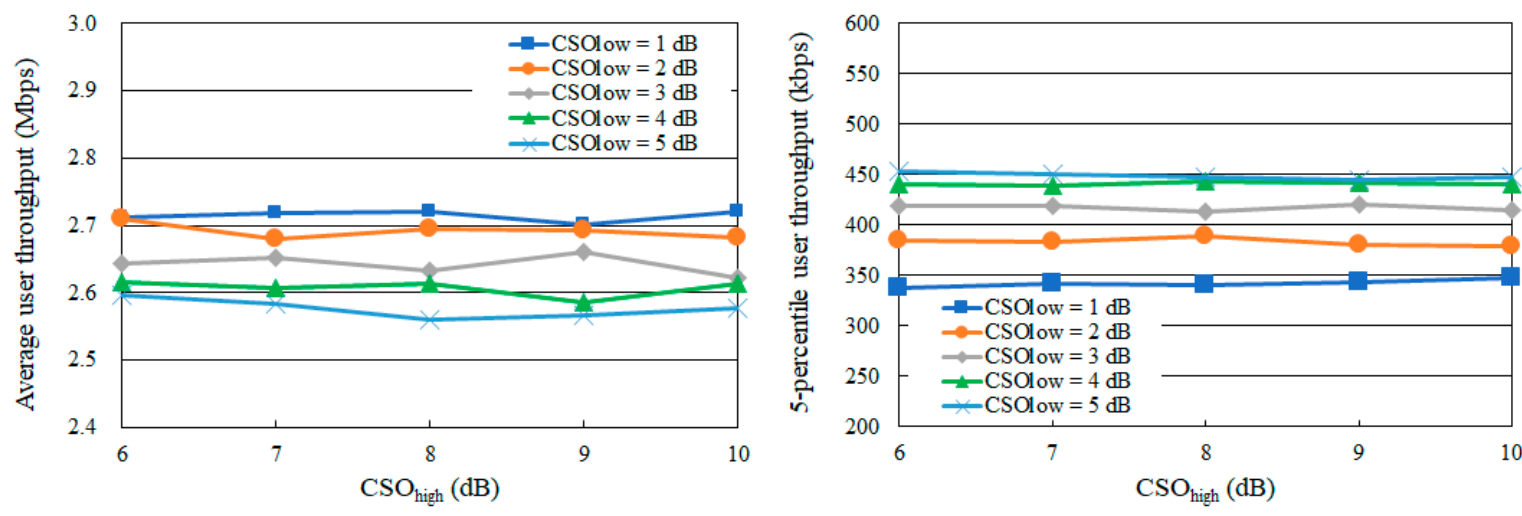

(a)
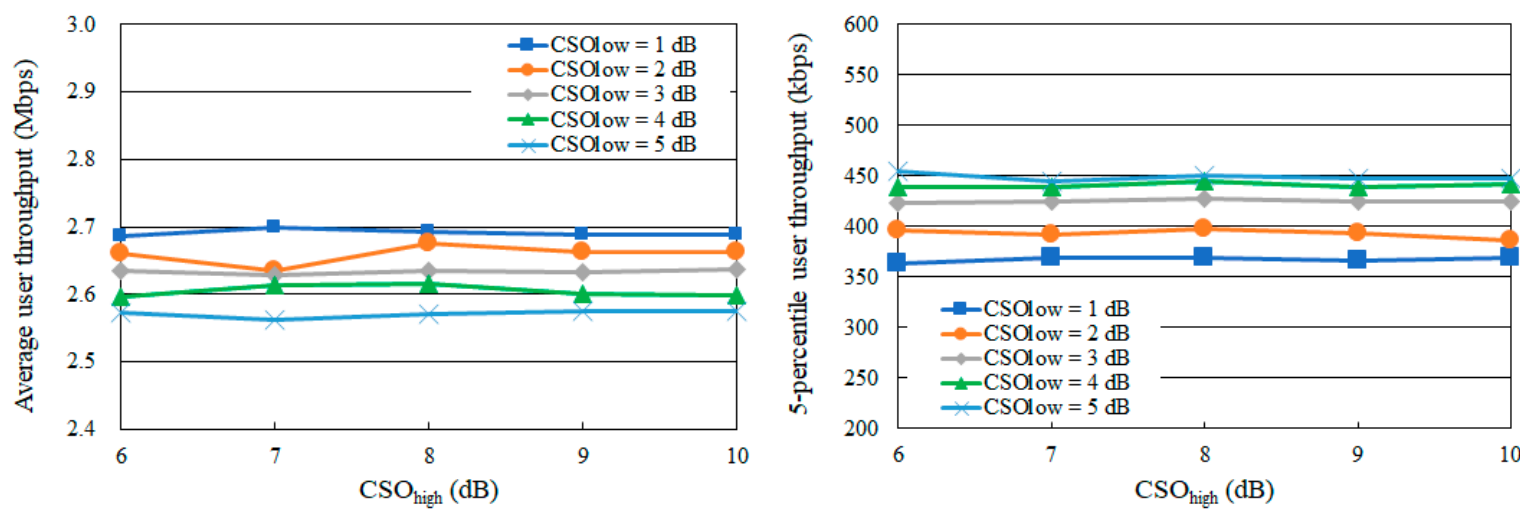

(b)
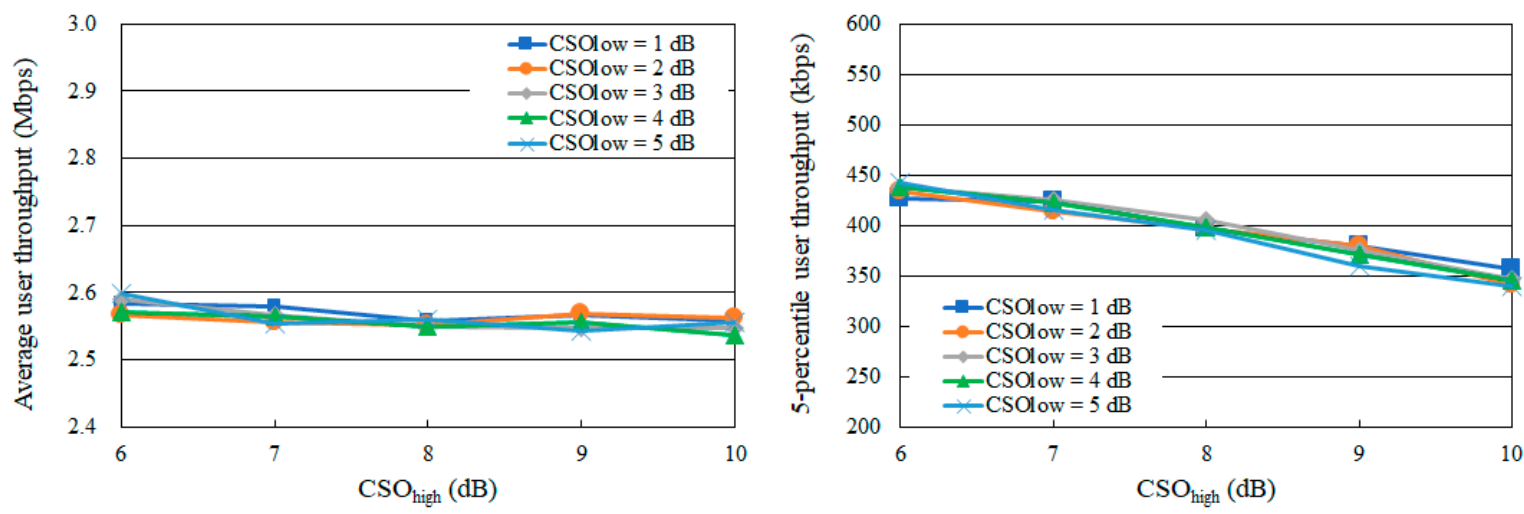

(c)
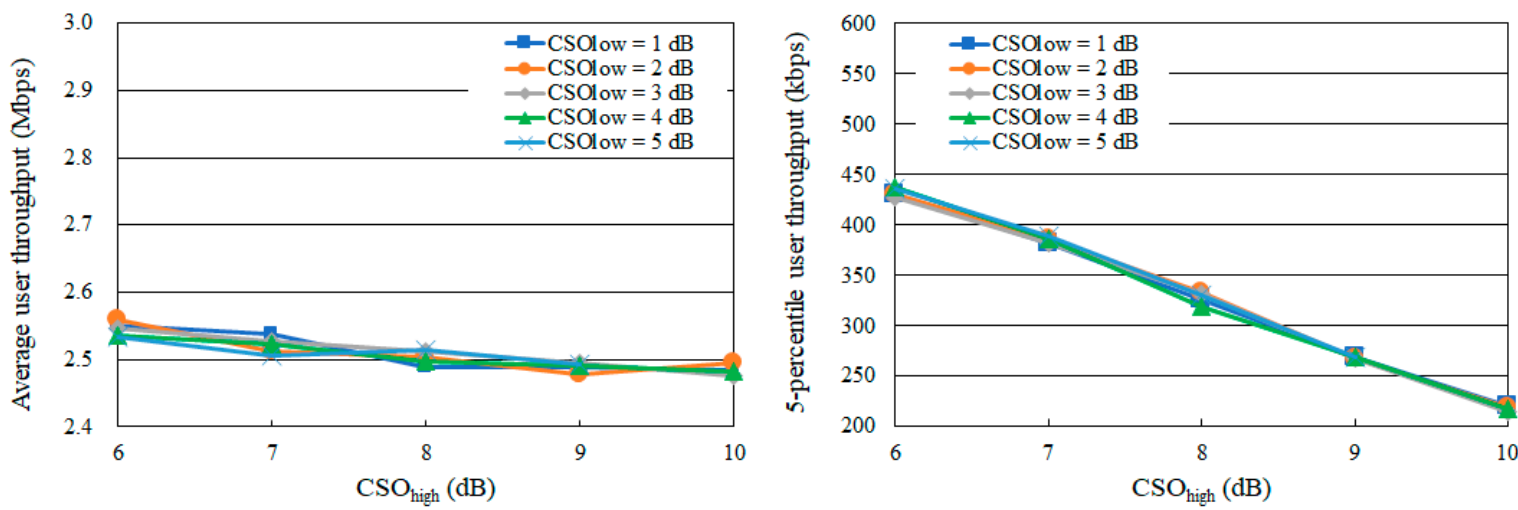

(d)

Figure 7. Average and 5-percentile user throughput versus $\mathrm{CSO}_{\text {high }}$ as a function of $\mathrm{CSO}_{\text {low }}(\mathbf{a})$ when $\alpha=0.2$; (b) $\alpha=0.4 ;$ (c) $\alpha=0.6 ;$ (d) $\alpha=0.8$. 


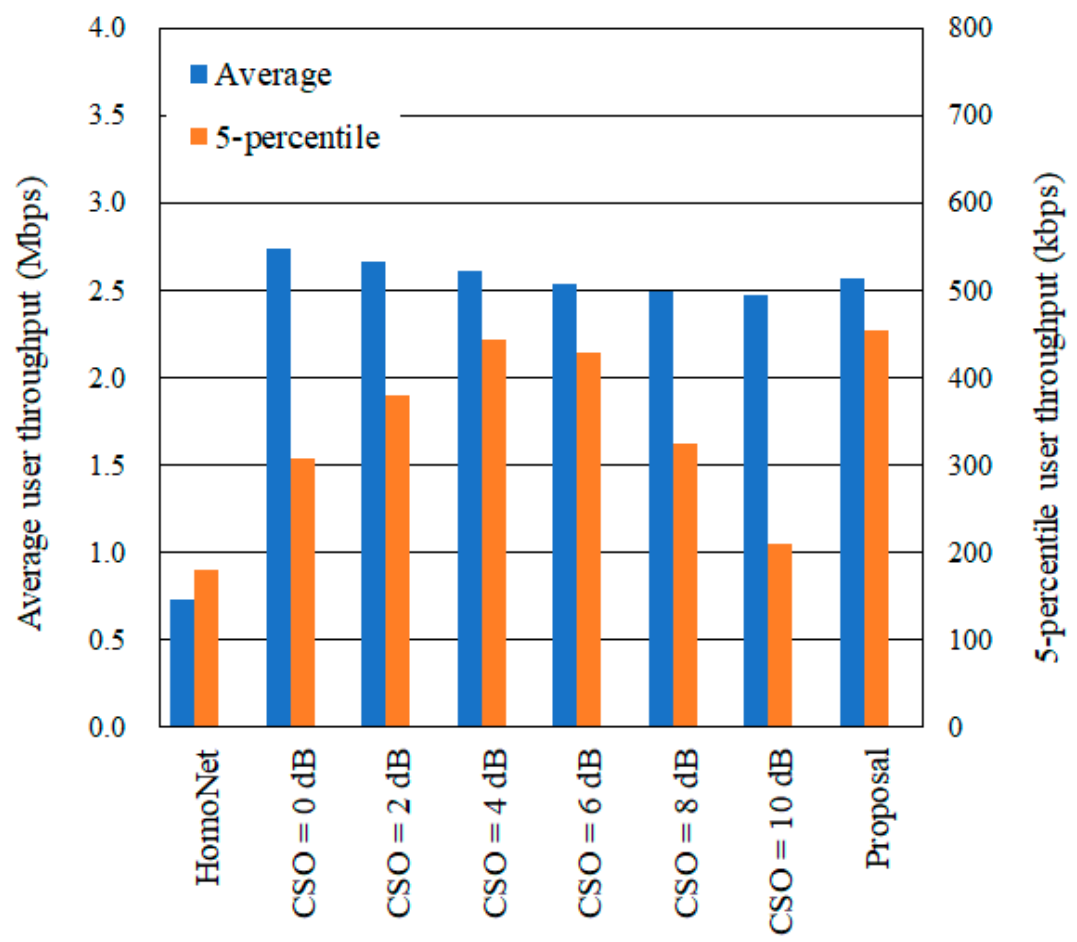

Figure 8. User throughput of adaptive control CRE compared with the conventional CRE and HomoNet in single-band HetNet.

\section{User Throughput in Multi-Band HetNet}

In this part, we evaluate the user throughput of adaptive control CRE in multi-band HetNet whose carrier frequencies used in the macro- and pico-eNBs are 2.0 and $3.4 \mathrm{GHz}$, respectively. Compared to single-band HetNet as shown in Section 3, multi-band HetNet causes interference only between picocells, i.e., there is no interference between macrocell and picocell. In terms of system bandwidth, we assumed that the bandwidth of the picocell is the same as that of the macrocell, as listed in Table 1. Other simulation conditions are same as for single-band HetNet. Like this, we discuss the user throughput of adaptive control CRE in multi-band HetNet under the same conditions of single-band HetNet except the carrier frequencies.

\subsection{Parameter Optimization in Multi-Band HetNet}

Figure $9 \mathrm{a}-\mathrm{d}$ shows the average and 5-percentile user throughput versus $\mathrm{CSO}_{\text {high }}$ as a function of $\mathrm{CSO}_{\text {low }}$ for $\alpha=0.2,0.4,0.6$, and 0.8 , respectively. The ranges of $\mathrm{CSO}_{\text {low }}$ and $\mathrm{CSO}_{\text {high }}$ are rather wide compared with those in single-band HetNet.

When $\alpha=0.2$, the 5-percentile user throughput worsens, although the average user throughput slightly increases compared with that at $\alpha=0$.When $\alpha=0.6$ or 0.8 , both 5-percentile and average user throughput worsens compared with those at $\alpha=0$. Thus, from the 5-percentile user throughput perspective, $\alpha=0.4$ provides best performance among $\alpha=0.2,0.4,0.6$, and 0.8 .

The behavior for average user throughput against $\mathrm{CSO}_{\text {low }}$ is the same as in single-band HetNet. When $\alpha=0.4$, the average user throughput hardly depends on $\mathrm{CSO}_{\text {high }}$. $\mathrm{CSO}_{\text {low }}$ of 0 or $3 \mathrm{~dB}$ provides better average user throughput than that of $\mathrm{CSO}_{\text {low }}$ of 6 or $9 \mathrm{~dB}$. Thus, considering both the average and 5-percentile user throughput, the parameters of $\alpha=0.4, \mathrm{CSO}_{\text {low }}=3 \mathrm{~dB}$, and $\mathrm{CSO}_{\text {high }}=12 \mathrm{~dB}$ are determined to be the best. 

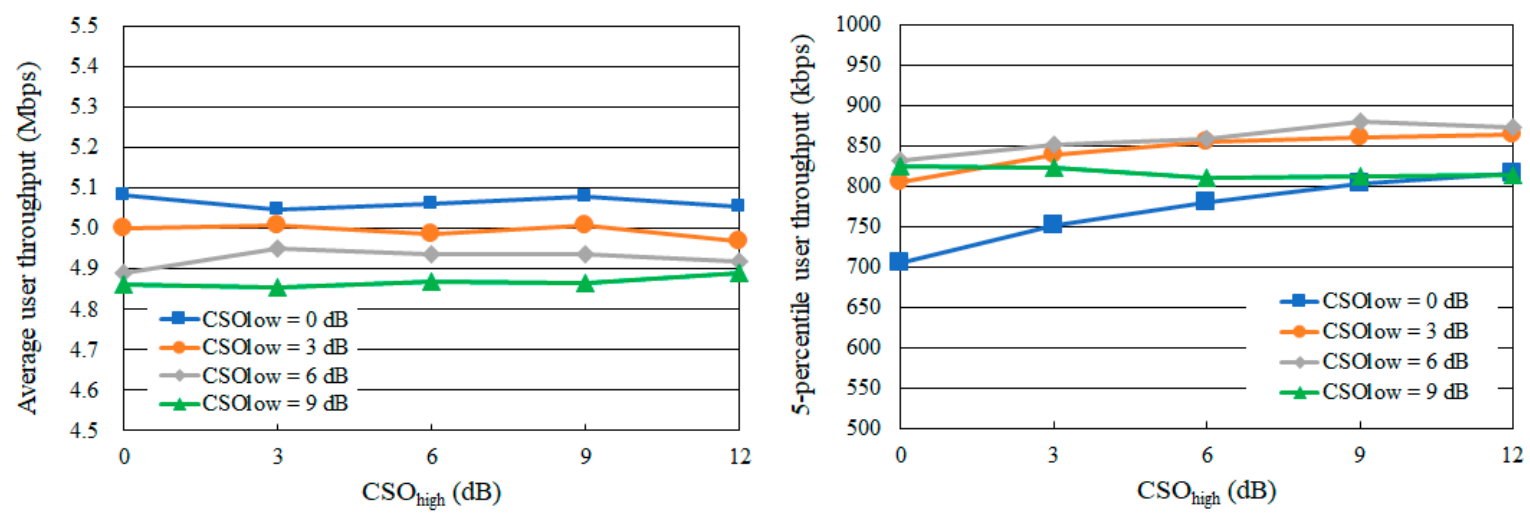

(a)
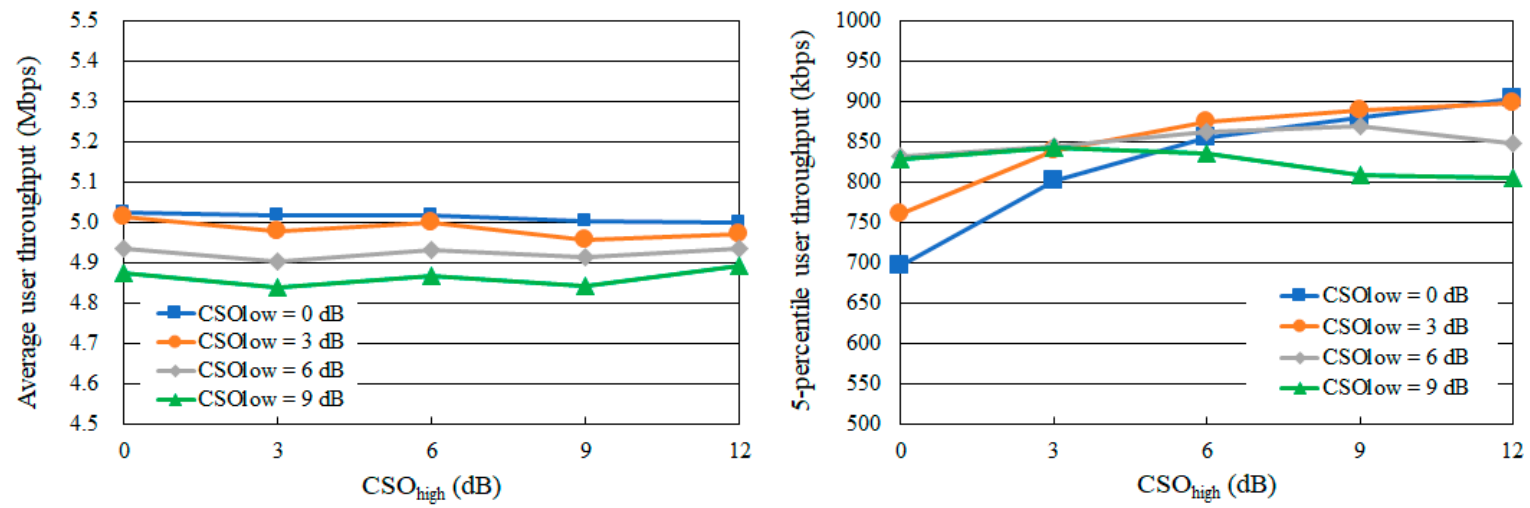

(b)
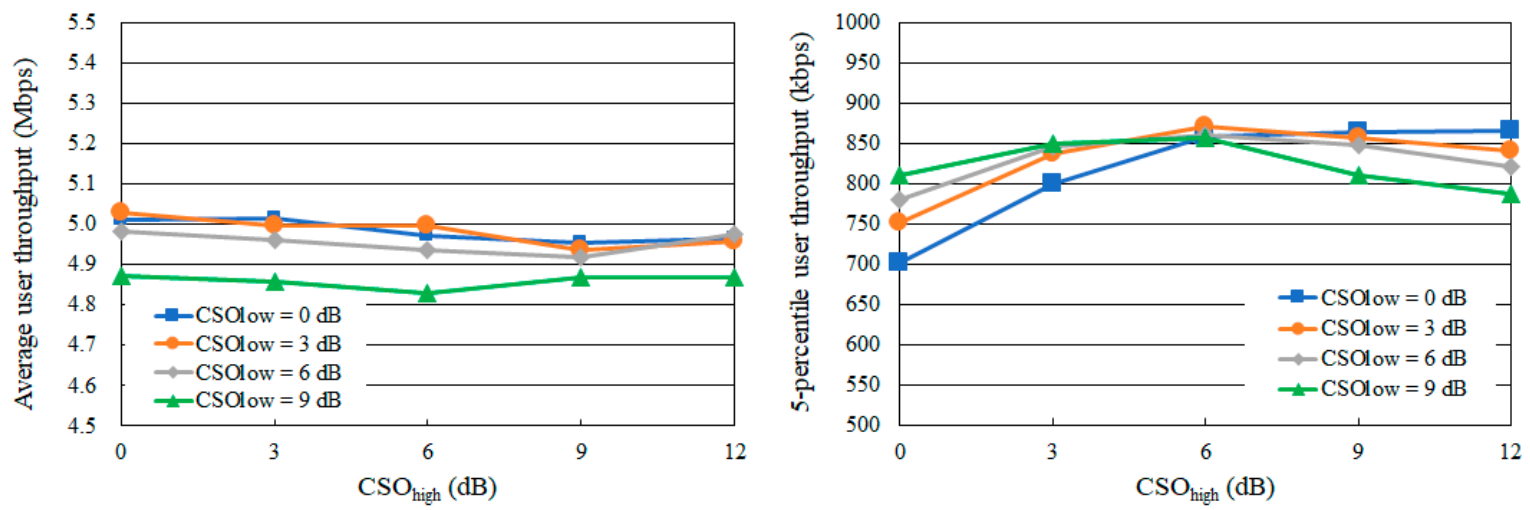

(c)
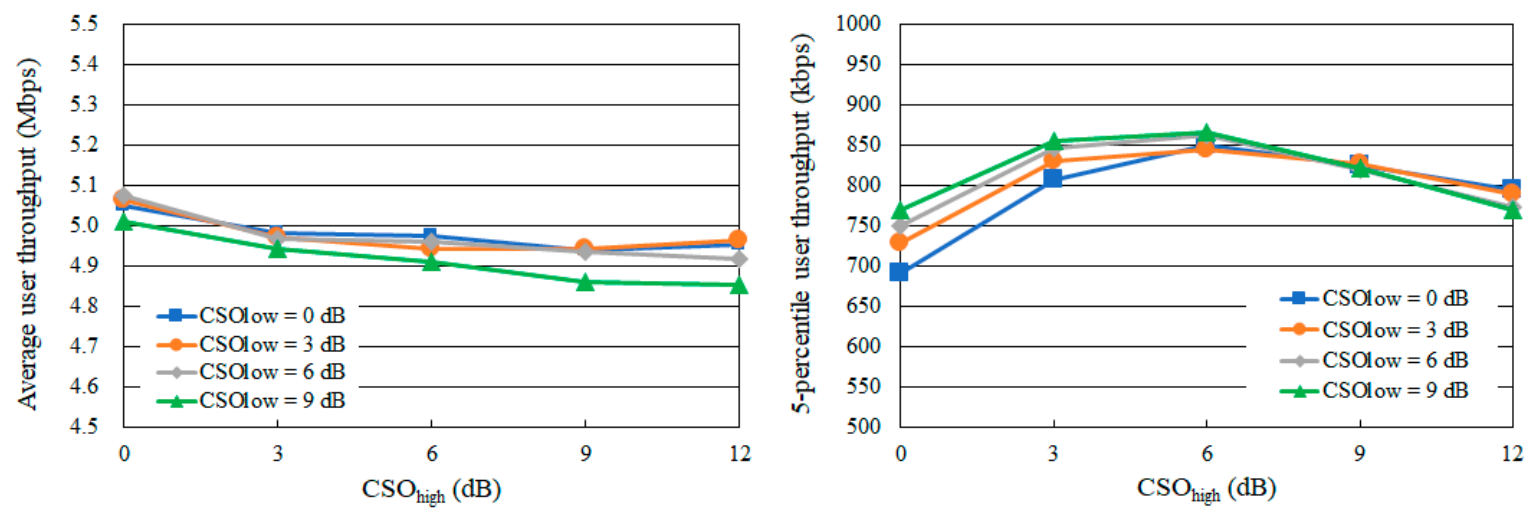

(d)

Figure 9. Average and 5-percentile user throughput versus $\mathrm{CSO}_{\text {high }}$ as a function of $\mathrm{CSO}_{\text {low }}$ (a) when $\alpha=0.2$; (b) $\alpha=0.4 ;$ (c) $\alpha=0.6$; (d) $\alpha=0.8$. 


\subsection{Comparison with Conventional Method}

Figure 10 shows the average and 5-percentile user throughput of the adaptive control CRE when using the optimal parameters determined as described in Section 4.1, i.e., $\alpha=0.4, \mathrm{CSO}_{\text {low }}=3 \mathrm{~dB}$, and $\mathrm{CSO}_{\text {high }}=12 \mathrm{~dB}$, compared with the conventional method and HomoNet.

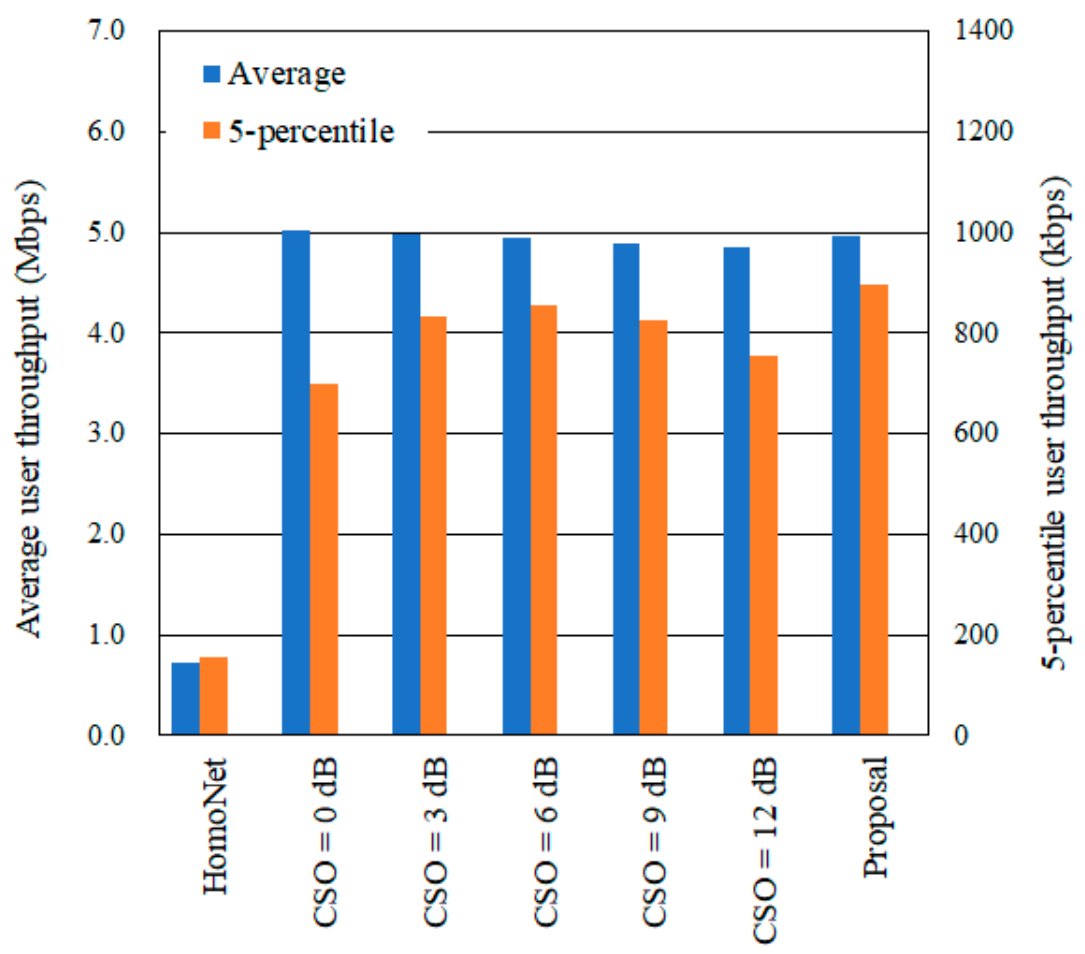

Figure 10. User throughput of adaptive control CRE compared with conventional CRE and HomoNet in multi-band HetNet.

For the conventional method, five kinds of fixed CSO are set: $\mathrm{CSO}=0,3,6,9$, and $12 \mathrm{~dB}$. The $\mathrm{CSO}_{\text {high }}$ can be greater than that in single-band HetNet because there is no interference between macrocell and picocells. The blue and orange bars show the average and 5-percentile user throughput, respectively.

The average user throughput decreases according to the increase in the CSO from 0 to $12 \mathrm{~dB}$ in the conventional CRE method. By increasing the CSO up to $6 \mathrm{~dB}$, the 5-percentile user throughput increases, although the average user throughput decreases. When CSO is more than $6 \mathrm{~dB}$, the conventional method degrades both the average and 5-percentile user throughput. The reason for this phenomenon is the same as in single-band HetNet. Adaptive control CRE can improve the 5-percentile user throughput by $8 \%$ while maintaining the average user throughput compared with those of conventional method (CSO of $6 \mathrm{~dB}$ ), as shown in Figure 10. Compared with a CSO of $0 \mathrm{~dB}$, the proposed method can improve 5-percentile user throughput by 1.4 times approximately, although the average user throughput slightly decreases.

As shown in Figures 8 and 10, the average user throughput slightly decreases as the CSO increases. However, the performance behavior in user throughput depends on the number of UE installations in the microcell or macro sector, and the type of UE distribution such as cluster distribution and uniform distribution. The system bandwidth of the picocell, i.e., the number of radio resources, also affects the performance behavior in user throughput. If the signal bandwidth of the picocell is wider than that of the microcell, the performance behavior in average user throughput is different from the results as shown in Figures 8 and 10. In ref. [29], it is shown that average user throughput increases as the CSO increases up to a certain value. In this paper, it is assumed that the signal bandwidth 
of picocell is the same as that of microcell, and the number of UE is relatively high, such as 30 per macro sector. Therefore, the average user throughput slightly decreases as the CSO increases.

\section{Conclusions}

In this paper, we have proposed a personal picocell scheme using an adaptive control CRE technique in which different CSOs are assigned to UE to form an optimal picocell for each UE.

First, we described the concept and architecture of the proposed personal picocell scheme in HetNets and compared with the conventional CRE, and we described the principle and connection sequence of the adaptive control CRE that uses two different CSOs: $\mathrm{CSO}_{\text {high }}$ and $\mathrm{CSO}_{\text {low }}$. Then, we presented the parameter optimization and user throughput of the adaptive control CRE for single-band and multi-band HetNets by using system-level computer simulations.

From these simulation results, we confirmed that the adaptive control CRE can improve the 5 -percentile user throughput by $5 \%$ and $8 \%$ while maintaining the average user throughput compared with conventional CRE for single-band HetNet and multi-band HetNet, respectively.

In our future work, we will investigate uplink user throughput and discuss the benefits of the proposed scheme. Furthermore, we will investigate user throughput for the increase of the system bandwidth of picocell, as well as the optimization for resource sharing between micro and picocells.

Author Contributions: Conceptualization, H.O. and K.F.; investigation, K.F., F.K. and H.O.; data curation, K.F. and F.K.; writing — original draft preparation, H.O.; writing-review and editing, H.O. and K.F.; visualization, K.F. and F.K.; supervision, H.O.; project administration, H.O.; funding acquisition, H.O. All authors have read and agreed to the published version of the manuscript.

Funding: This research was funded by JSPS KAKENHI Grant Number JP18K11277, Grant-in-Aid for Scientific Research (C).

Acknowledgments: The authors would like to thank K. Kikuchi who is graduates of Kogakuin University for their contributions to construct link-level simulations.

Conflicts of Interest: The authors declare no conflict of interest.

\section{References}

1. Andrews, J.G.; Buzzi, S.; Choi, W.; Hanly, S.V.; Lozano, A.E.; Soong, A.C.K.; Zhang, J.C. What Will 5G Be? IEEE J. Sel. Areas Commun. 2014, 32, 1065-1082. [CrossRef]

2. Soldani, D.; Manzalini, A. Horizon 2020 and Beyond: On the 5G Operating System for a True Digital Society. IEEE Veh. Technol. Mag. 2015, 10, 32-42. [CrossRef]

3. Nakamura, T.; Benjebbour, A.; Kishiyama, Y.; Suyama, S.; Imai, T. 5G Radio Access: Requirements, Concept and Experimental Trials. IEICE Trans. Commun. 2015, E98-B, 1397-1406. [CrossRef]

4. Bogale, T.E.; Le, L.B. Massive MIMO and mmWave for 5G Wireless HetNet: Potential Benefits and Challenges. IEEE Veh. Technol. Mag. 2016, 11, 64-75. [CrossRef]

5. Okumura, Y.; Suyama, S.; Mashino, J.; Muraoka, K. Recent Activities of 5G Experimental Trials on Massive MIMO Technologies and 5G System Trials Toward New Services Creation. IEICE Trans. Commun. 2019, E102-B, 1352-1362. [CrossRef]

6. Ishii, H.; Kishiyama, Y.; Takahashi, H. A novel architecture for LTE-B:C-plane/U-plane split and Phantom Cell concept. In Proceedings of the 2012 IEEE Globecom Workshops; Institute of Electrical and Electronics Engineers (IEEE), Anaheim, CA, USA, 3-7 December 2012; pp. 624-630. [CrossRef]

7. Ghosh, A.; Ratasuk, R.; Mondal, B.; Mangalvedhe, N.; Thomas, T. LTE-advanced: Next-generation wireless broadband technology [Invited Paper. IEEE Wirel. Commun. 2010, 17, 10-22. [CrossRef]

8. Dahlman, E.; Dimou, K.; Parkvall, S.; Tullberg, H. Future wireless access small cells and heterogeneous deployments. In Proceedings of the ICT 2013 Institute of Electrical and Electronics Engineers (IEEE), Casablanca, Morocco, 6-8 May 2013; pp. 1-5. [CrossRef]

9. Deb, S.; Monogioudis, P.; Miernik, J.; Seymour, J.P. Algorithms for Enhanced Inter-Cell Interference Coordination (eICIC) in LTE HetNets. IEEE/ACM Trans. Netw. 2013, 22, 137-150. [CrossRef]

10. Chi, H.R.; Radwan, A. Multi-Objective Optimization of Green Small Cell Allocation for IoT Applications in Smart City. IEEE Access 2020, 8, 101903-101914. [CrossRef] 
11. Dghais, W.; Souilem, M.; Chi, H.R.; Radwan, A.; Taha, A.-E.M. Dynamic Clustering for Power Effective Small Cell Deployment in HetNet 5G Networks. In Proceedings of the ICC 2020-2020 IEEE International Conference on Communications (ICC); Institute of Electrical and Electronics Engineers (IEEE), Dublin, Ireland, 7-11 June 2020; pp. 1-5. [CrossRef]

12. Potential Performance of Range Expansion in Macro-Pico Deployment (R1-104355); TSG RAN WG1; 3GPP: Madrid, Spain, 2010.

13. Tian, P.; Tian, H.; Zhu, J.; Chen, L.; She, X. An adaptive bias configuration strategy for range extension in LTE-advanced heterogeneous networks. In Proceedings of the IET International Conference on Communication Technology and Application (ICCTA 2011); Institution of Engineering and Technology (IET), Beijing, China, 14-16 October 2011; pp. 336-340. [CrossRef]

14. Guvenc, I. Capacity and Fairness Analysis of Heterogeneous Networks with Range Expansion and Interference Coordination. IEEE Commun. Lett. 2011, 15, 1084-1087. [CrossRef]

15. Kikuchi, K.; Otsuka, H. Proposal of adaptive control CRE in heterogeneous networks. In Proceedings of the 2012 IEEE 23rd International Symposium on Personal, Indoor and Mobile Radio Communications-(PIMRC); Institute of Electrical and Electronics Engineers (IEEE), Sydney, NSW, Australia, 9-12 September 2012; pp. 910-914. [CrossRef]

16. Kikuchi, K.; Otsuka, H. Parameter optimization for adaptive control CRE in HetNet. In Proceedings of the 2013 IEEE 24th Annual International Symposium on Personal, Indoor and Mobile Radio Communications (PIMRC); Institute of Electrical and Electronics Engineers (IEEE), London, UK, 8-11 September 2013; pp. 3334-3338. [CrossRef]

17. Nakazawa, S.; Matsuoka, S.; Otsuka, H. Adaptive control CRE and its throughput performance in HetNet. IEICE Commun. Express 2015, 4, 117-122. [CrossRef]

18. Nakazawa, S.; Naganuma, N.; Otsuka, H. Enhanced adaptive control CRE in heterogeneous networks. In Proceedings of the 2017 14th IEEE Annual Consumer Communications \& Networking Conference (CCNC); Institute of Electrical and Electronics Engineers (IEEE), Las Vegas, NV, USA, 8-11 January 2017; pp. 645-646. [CrossRef]

19. Naganuma, N.; Nakazawa, S.; Suyama, S.; Okumura, Y.; Otsuka, H. Adaptive control CRE technique for eICIC in HetNet. In Proceedings of the 2016 Eighth International Conference on Ubiquitous and Future Networks (ICUFN); Institute of Electrical and Electronics Engineers (IEEE), Vienna, Austria, 5-8 July 2016; pp. 4-6. [CrossRef]

20. Fujisawa, K.; Kemmochi, F.; Otsuka, H. Personal-Cell Scheme Using Adaptive Control CRE for Multicarrier HetNets. In Proceedings of the 2019 IEEE 90th Vehicular Technology Conference (VTC2019-Fall); Institute of Electrical and Electronics Engineers (IEEE), Honolulu, HI, USA, 22-25 September 2019; pp. 1-5. [CrossRef]

21. Mu, Q.; Liu, L.; Chen, L.; Jiang, Y. CQI table design to support 256 QAM in small cell environment. In Proceedings of the 2013 International Conference on Wireless Communications and Signal Processing; Institute of Electrical and Electronics Engineers (IEEE), Hangzhou, China, 24-26 October 2013; pp. 1-5. [CrossRef]

22. Iwamoto, M.; Matsuoka, S.; Iwasaki, H.; Otsuka, H. Transmission performance of OFDM with 1024-QAM in the presence of EVM degradation. In Proceedings of the 2014 IEEE Asia Pacific Conference on Wireless and Mobile; Institute of Electrical and Electronics Engineers (IEEE), Bali, Indonesia, 28-30 August 2014; pp. $12-16$. [CrossRef]

23. Ota, T.; Nakamura, M.; Otsuka, H. Performance evaluation of OFDM-based 256- and 1024-QAM in multipath fading propagation conditions. In Proceedings of the 2017 Ninth International Conference on Ubiquitous and Future Networks (ICUFN); Institute of Electrical and Electronics Engineers (IEEE), Milan, Italy, 4-7 July 2017; pp. 554-556. [CrossRef]

24. Naganuma, N.; Osawa, K.; Mashino, J.; Suyama, S.; Otsuka, H. Proposal of CQI table switching control method corresponding to 256-QAM for CRE in HetNet. In Proceedings of the 2018 International Conference on Information Networking (ICOIN); Institute of Electrical and Electronics Engineers (IEEE), Chiang Mai, Thailand, 10-12 January 2018; pp. 331-335. [CrossRef]

25. ITU Report, M.2135-1. Guidelines for Evaluation of Radio Interface Technologies for IMT-Advanced; International Telecommunication Union: Geneva, Switzerland, 2009.

26. Evolved Universal Terrestrial Radio Access (E-UTRA); Introduction of 1024 Quadrature Amplitude Modulation (QAM) in LTE downlink (Release 15); Technical report No. 36.783; 3GPP: Valbonne, France, 2017. 
27. Tian, R.; Senda, K.; Ota, T.; Otsuka, H. Transmission performance of OFDM-based 1024-QAM in multipath fading conditions. IEICE Commun. Express 2018, 7, 272-277. [CrossRef]

28. Otsuka, H.; Tian, R.; Senda, K. Transmission Performance of an OFDM-Based Higher-Order Modulation Scheme in Multipath Fading Channels. J. Sens. Actuator Netw. 2019, 8, 19. [CrossRef]

29. Kemmochi, F.; Fujisawa, K.; Otsuka, H. Potential Design for Modulation and Coding Scheme in mmWave Multicarrier HetNets. In Proceedings of the 2019 IEEE 90th Vehicular Technology Conference (VTC2019-Fall); Institute of Electrical and Electronics Engineers (IEEE), Honolulu, HI, USA, 22-25 September 2019; pp. 3-12. [CrossRef]

Publisher's Note: MDPI stays neutral with regard to jurisdictional claims in published maps and institutional affiliations.

(C) 2020 by the authors. Licensee MDPI, Basel, Switzerland. This article is an open access article distributed under the terms and conditions of the Creative Commons Attribution (CC BY) license (http://creativecommons.org/licenses/by/4.0/). 